\title{
155 Acute maxillary sinusitis
}

A The infection is of dental origin in $1 \%$ of cases.

$B$ Tenderness is usually localized over the sinus.

C X-rays are of no value in the acute phase.

D Antral washouts should be performed as soon as possible, to establish the diagnosis and commence treatment.

E Toothache precedes sinusitis in all cases of apical abscess.

\section{Acute frontal sinusitis}

A The frontal sinus is usually affected alone.

B Pain is typically worse in the morning.

C Discharge is seen in the inferior meatus, where the frontonasal duct opens.

D Treatment is to cannulate the frontonasal duct.

E Trephining the orbital roof should be avoided because of the danger of spreading infection to the eye.

\section{Acute sphenoidal sinusitis}

A Is not uncommon.

B The posterior ethmoidal cells are involved in most cases.

C Pain may simulate acute mastoiditis.

D Discharge is seen at the back of the nose.

E The sinus can be punctured and washed out transnasally.

\section{Treatment of chronic maxillary sinusitis}

A Medical treatment is useless; surgery is nearly always required.

B Antral washouts should be performed daily for 3 weeks in the first instance.

C Polyps in the sinus can be removed most effectively via intranasal antrostomy.

D Caldwell-Luc operation involves enlarging the natural ostium to allow free drainage.

E A sublabial antrostomy is contraindicated in the presence of irreversible pathological change in the mucosa. 